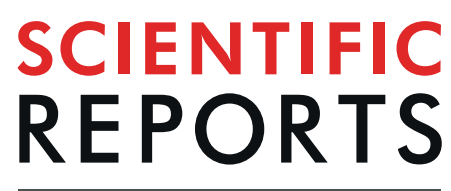

natureresearch

\title{
Immunity-related genes and signaling pathways under hypoxic stresses in Haliotis diversicolor: a transcriptome analysis
}

\author{
Yulong Sun ${ }^{1,3}$, Xin Zhang ${ }^{1,3}$, Yilei Wang $\mathbb{1 0}^{2 *}$, Robert Day ${ }^{4}$, Huiping Yang ${ }^{5}$ \& Ziping Zhang $\mathbb{D}^{1,2^{*}}$
}

Due to increased temperatures and aquaculture density, thermal and hypoxia stresses have become serious problems for the aquaculture of abalone Haliotis diversicolor. Stresses lead to immunosuppression, which can cause severe negative impacts on aquaculture farms. To study the mechanism of immunosuppression after hypoxia stress and bacterial challenge, transcriptomes of $H$. diversicolor hemocytes involved in immunity were profiled. A total of 307,395,572 clean reads were generated and assembled into 99,774 unigenes. KEGG analysis indicated that 225 unigenes with immunologic function were mapped into immune-related pathways. Expression of 41 unigenes measured by quantitative real-time PCR (qRT-PCR) showed consistent results with that of transcriptome analysis. When exposure challenge of Vibrio parahaemolyticus, it is indicated that the PI3K-AKT, MAPK, NF- $K B$ and P53 signal pathways were involved in the hypoxia-induced immunosuppression of $H$. diversicolor. Furthermore, when the AKT gene (HdAKT) was inhibited by double-stranded RNA (dsRNA), expression levels of HdAKT was lower than the blank and control group in hemocytes at $4 \mathrm{~h}, 12 \mathrm{~h}$ and $24 \mathrm{~h}(p<0.05)$.

Haliotis diversicolor (named 'small abalone' in China) is one of the most commercially important aquacultured abalone species in the coastal provinces of southern China. The abalone aquaculture industry has been threatened by deteriorating environmental conditions and infectious diseases, especially in hot summers ${ }^{1}$. High temperature of seawater could result in low oxygen levels, and subsequently caused changes in respiration and metabolism of marine benthos ${ }^{2}$. Additionally, outbreaks of diseases such as the disease caused by major pathogen Vibrio parahaemolyticus, could occur in hot summers and cause mass mortality of cultured abalone $\mathrm{e}^{3,4}$. Therefore, understanding of the effects of high temperature and disease on immune functions of $H$. diversicolor is needed for improvement of abalone aquaculture.

Under environmental and bacterial challenges, several immune related genes were cloned in $H$. diversicolor and the activation of these gene were investigated ${ }^{5-14}$. For example, the macrophage expressed gene ${ }^{7}$, insulin-like growth factor binding protein $7^{8}$, interleukin-1 receptor-associated kinase $4^{9}$, macrophage migration inhibitory factor $^{5}$, and genes correlated with the NF- $\kappa B$ signaling pathway ${ }^{10}$, HIF signaling pathway ${ }^{13}$, toll-like receptor signaling pathway ${ }^{9}$ and the PI3K-AKT signal pathway ${ }^{14}$ have been cloned and characterized from $H$. diversicolor (see Additional file 1: Table S1 for the acronyms used in this paper). Particularly, it has been proved that the NF- $\kappa \mathrm{B}$ signaling pathway-related genes, heat shock responsive genes, and HIF signaling pathway-related genes were significantly up-regulated in $H$. diversicolor after thermal or hypoxia stress ${ }^{10,11,13}$. Furthermore, genes related to the PI3K-AKT signal pathway were significantly down-regulated and physiological responses were affected, leading to immunosuppression when $H$. diversicolor was exposed to multiple stresses ${ }^{14}$.Using an assay of physiological and biochemical parameters of hemocytes, immunosuppression was found to be caused by high temperature ${ }^{15}$.

${ }^{1}$ College of Animal Science, Fujian Agriculture and Forestry University, Fuzhou, 350002, P.R. China. ${ }^{2}$ Fisheries College, Jimei University, Xiamen, 361021, China. ${ }^{3}$ Key Laboratory of Marine Biotechnology of Fujian Province, Institute of Oceanology, Fujian Agriculture and Forestry University, Fuzhou, 350002, P.R. China. ${ }^{4}$ School of Biosciences, University of Melbourne, Parkville, Victoria, 3010, Australia. ${ }^{5}$ School of Forest Resources and Conservation, IFAS, University of Florida, 7922 NW 71st Street, Gainesville, FL, 32615, USA. *email: ylwang@jmu.edu.cn; zhangziping@ hotmail.com 


\begin{tabular}{|l|l|l|l|l|}
\hline Sample & Before Filter Reads Num & After Filter Reads Num(\%) & GC\% & $\% \geq \mathbf{Q 3 0}$ \\
\hline BC-0h & 24354936 & $23778916(97.63 \%)$ & $42.69 \%$ & $95.85 \%$ \\
\hline NC-4h & 20829374 & $20344880(97.67 \%)$ & $43.41 \%$ & $95.61 \%$ \\
\hline BC-4h & 20899430 & $20489268(98.04 \%)$ & $42.96 \%$ & $95.91 \%$ \\
\hline NC-12h & 21340802 & $20737464(97.17 \%)$ & $42.96 \%$ & $94.66 \%$ \\
\hline BC-12h & 23774786 & $23119858(97.25 \%)$ & $42.70 \%$ & $94.91 \%$ \\
\hline NC-24h & 21203460 & $20694196(97.6 \%)$ & $45.67 \%$ & $95.41 \%$ \\
\hline BC-24h & 21677186 & $21209236(97.84 \%)$ & $42.77 \%$ & $95.78 \%$ \\
\hline NC-48h & 20800322 & $20154168(96.89 \%)$ & $43.06 \%$ & $95.03 \%$ \\
\hline BC-48h & 19556898 & $18974014(97.02 \%)$ & $43.12 \%$ & $94.83 \%$ \\
\hline HS-0h & 24693066 & $23969486(97.07 \%)$ & $44.50 \%$ & $94.43 \%$ \\
\hline HS-4h & 19287670 & $18748906(97.21 \%)$ & $43.27 \%$ & $95.00 \%$ \\
\hline HS-12h & 20653144 & $20167554(97.65 \%)$ & $42.83 \%$ & $95.85 \%$ \\
\hline HS-24h & 29409970 & $28492076(96.88 \%)$ & $42.75 \%$ & $94.76 \%$ \\
\hline HS-48h & 27383712 & $26515550(96.83 \%)$ & $42.91 \%$ & $94.80 \%$ \\
\hline
\end{tabular}

Table 1. Assembly statistics of the transcriptome of the $14 \mathrm{cDNA}$ libraries. The $14 \mathrm{cDNA}$ libraries (BC-0h, BC-4h, BC-12h, BC-24h, BC-48h, NC-4h, NC-12h, NC-24h, NC-48h, HS-0h, HS-4h, HS-12h, HS-24h and HS-48h) were assembled by using the Trinity software package. BC: Blank control group, normal conditions; NC: Control group, bacterial challenge under normal conditions; HS: Experimental group, hypoxia stress and bacterial challenge.

So far, many research has demonstrated that environment stresses can lead to immunosuppression in vertebrates and invertebrates ${ }^{16-22}$.

Our published results mentioned above have also confirmed that the immune regulatory mechanisms of $H$. diversicolor are activated after exposure to thermal and hypoxia stresses and bacterial challenge. Thus, multiple stressors may lead to several changes including immunosuppression in abalone. Unfortunately, our knowledge of the immune response and signaling pathways that respond to hypoxia, thermal stress and bacterial pathogens in $H$. diversicolor is still fragmentary.

Development of DNA sequencing technology, such as the next generation sequencing (NGS), provides high-throughput tools to analyze the expression of various genes, discover novel transcripts, and identify differentially expressed genes (DEGs). NGS also provides large sequencing datasets at an affordable price ${ }^{23,24}$. Using the NGS technology, transcriptome analyses of Haliotis species and many other aquaculture mollusks have been performed and published in the recent five years ${ }^{25-41}$. These studies included gene expression during the early development of $H$. diversicolor, transcriptome profiles of wild and cultured populations of Haliotis midae ${ }^{31}$ and expression and putative function of heat shock protein 70 (HSP70) genes of Haliotis laevigata under environmental stress ${ }^{27}$. Previous transcriptome analysis showed that several innate immunity related pathways, such as NF- $\kappa B$ signaling pathway, Toll-like receptor signaling pathway, and PI3K-AKT signaling pathway are involved in immune response to environmental stress without pathogen infection in $H$. diversicolor ${ }^{42}$. These studies led to discovery of many candidate genes and provided valuable information to understand the biological characteristics of marine mollusks. However, detailed cellular and molecular mechanisms were not clearly documented yet.

In this study, the goal was to analyze the transcriptome changes in the hemocytes of $H$. diversicolor after exposure to bacterial challenge with or without hypoxia to reveal the molecular mechanisms and the immune responses. The objectives were to: (1) assemble transcriptome profile through RNAseq; (2) identify the differentially expressed genes in response to bacterial challenges with hypoxia or without hypoxia; (3) validate immune related genes through qRT-PCR; (4) construct the gene networks of the signaling pathways related to immunology, and (5) evaluate the interaction among the PI3K-AKT signaling pathway-related genes through RNA inhibition of $H d A K T$. This study provided a preliminary understanding of the molecular mechanisms in H. diversicolor in immunological response to bacterial challenges and hypoxia stresses.

\section{Results}

Sequencing and De novo assembly. Fourteen cDNA libraries, including BC-0h/NC-0h, BC-4h, BC-12h, BC-24h, BC-48h, NC-4h, NC-12h, NC-24h, NC-48h, HS-0h, HS-4h, HS-12h, HS-24h and HS-48h, were constructed using Illumina HiseqTM2000 paired-end sequencing technology. The average mean value of the Q30 percentage was $95.20 \%$. After removing the adapter sequences, ambiguous nucleotides, and low-quality sequences, a total of $307,395,572$ clean reads were generated through Illumina sequencing, containing 23,778,916 reads for the blank group (BC), 20,344,880 reads for the normal condition (NC), and 23,969,486 reads for the hypoxia stress group (HS). The average percentages of GC content for the clean reads were $42.69 \%, 43.41 \%$ and $44.50 \%$ for the BC, NC and HS (Table 1 ).

The clean reads were assembled into 99,774 transcripts with a total length of 7,752,922 nucleotides. The average length of a transcript was $768.27 \mathrm{bp}$ and the N50 length (the transcript length where the cumulative length of longer transcripts is $50 \%$ of the total) was 1,414. The size-distribution of these unigenes is shown in Fig. S1. 


\begin{tabular}{|l|l|l|l|l|}
\hline Pair & $\begin{array}{l}\text { Cellular } \\
\text { component } \\
\text { (CC) }\end{array}$ & $\begin{array}{l}\text { Molecular } \\
\text { function } \\
\text { (MF) }\end{array}$ & $\begin{array}{l}\text { Biological } \\
\text { process } \\
\text { (BP) }\end{array}$ & $\begin{array}{l}\text { All } \\
\text { DEGs }\end{array}$ \\
\hline BC-4h-VS-NC-4h Up-regulated & 1684 & 1839 & 3849 & 7372 \\
\hline BC-4h-VS-NC-4h Down-regulated & 628 & 607 & 1049 & 2284 \\
\hline BC-12h-VS-NC-12h Up-regulated & 338 & 443 & 955 & 1736 \\
\hline BC-12h-VS-NC-12h Down-regulated & 838 & 1016 & 1800 & 3654 \\
\hline BC-24h-VS-NC-24h Up-regulated & 1910 & 2317 & 5035 & 9262 \\
\hline BC-24h-VS-NC-24h Down-regulated & 1112 & 698 & 1375 & 3185 \\
\hline BC-48h-VS-NC-48h Up-regulated & 729 & 724 & 1330 & 2783 \\
\hline BC-48h-VS-NC-48h Down-regulated & 483 & 515 & 1072 & 2070 \\
\hline NC-0h-VS-HS-0h Up-regulated & 2035 & 2480 & 5130 & 9645 \\
\hline NC-0h-VS-HS-0h Down-regulated & 1569 & 857 & 1678 & 4104 \\
\hline NC-4h-VS-HS-4h Up-regulated & 619 & 749 & 1431 & 2799 \\
\hline NC-4h-VS-HS-4h Down-regulated & 913 & 659 & 1357 & 2929 \\
\hline NC-12h-VS-HS-12h Up-regulated & 1853 & 1991 & 3995 & 7839 \\
\hline NC-12h-VS-HS-12h Down-regulated & 564 & 536 & 882 & 1982 \\
\hline NC-24h-VS-HS-24h Up-regulated & 1714 & 1851 & 3445 & 7010 \\
\hline NC-24h-VS-HS-24h Down-regulated & 1017 & 779 & 1561 & 3357 \\
\hline NC-48h-VS-HS-48h Up-regulated & 637 & 551 & 1077 & 2265 \\
\hline NC-48h-VS-HS-48h Down-regulated & 2730 & 2303 & 4356 & 9389 \\
\hline
\end{tabular}

Table 2. Counts of the number of DEGs based on comparisons between treatment groups at each sampling time. Sampling time including NC/BC, HS/NC at $4 \mathrm{~h}, 12 \mathrm{~h}, 24 \mathrm{~h}$ and $48 \mathrm{~h}$. The comparison of BC and NC (NC/ BC) at $24 \mathrm{~h}$ produced most DEGs $(12,447)$. BC: Blank control group, normal conditions; NC: Control group, bacterial challenge under normal conditions; HS: Experimental group, hypoxia stress and bacterial challenge.

Functional annotation and classification. Unigenes annotated using the BLAST ${ }^{43}$ algorithm against the $\mathrm{Nr}$, Swiss-Prot, GO, COG and $\mathrm{KEGG}^{44-48}$ databases were illustrated by a Venn diagram in Fig. S2 (Fig. S2 cited from the earlier publication of Zhang et al. ${ }^{42}$, the data of sequencing and De novo assembly used in this paper and article of Zhang et al. were from the same sequencing project. Therefore, the De novo assembly result is the same, but RNA-seq data for expression analysis are different).

Classification of the 99,774 transcripts in GO functions using the Blast2GO software is illustrated in Fig. S3 (Fig. S3 cited from the earlier publication of Zhang et $a l .{ }^{42}$ ). The biological process category had 21 subcategories with cellular processes and metabolic processes as the major ones. The category of cellular component contained 16 subcategories with cell and cell part as the dominant ones. The molecular function contained 12 subcategories with binding and catalytic activity having the largest number of unigenes.

The comparison of different treatment groups revealed that the largest number of differentially expressed genes (DEGs) was at $24 \mathrm{~h}$ between the blank group and the normal condition (Table 2). The GO functional classifications of the DEGs at each sampling time are shown in Additional file 2: Fig. S4A-E.

The number of 10,840 unigenes were assigned significant matches to the KEGG database. A number were assigned to the pathways of immune system and environmental adaptation. The gene function enrichment analysis (Fig. 1) indicated that 225 unigenes were annotated with immunologic function and mapped into several immune-related pathways, including the MAPK, P38, NF- $\kappa$ B, HIF, and PI3K-AKT signaling pathway (Additional file 1: Table S2)

Analysis of expression patterns of immune - related genes. The expression of 225 immune-related genes revealed that the expressions of 90 unigenes were related to the PI3K-AKT signal pathway, 62 unigenes were related to the MAPK signal pathway, 20 were related to the NF- $\kappa B$ signal pathway, 24 to the P53 signal pathway, 18 to the HIF signal pathway, 7 to the Toll-like receptor signaling pathway and 4 unigenes were heat shock responsive.

Further, The RPKM relative expression in the control group (NC) was compared with the blank group (BC) and the fold change between groups $(\mathrm{NC} / \mathrm{BC})$ was calculated to show the change of gene expression as a result of the infection by the bacteria. The expression significantly increased at $4 \mathrm{~h}$ after the infection, but there was a significant decrease at $24 \mathrm{~h}$ and $48 \mathrm{~h}$ after infection (Additional file 2: Fig. S5). The RPKM relative expression between groups (HS/NC) was calculated. Most of these genes showed a significant decrease in expression after $4 \mathrm{~h}$ and $48 \mathrm{~h}$ of infection, only a few gene expressions were found to be up-regulated at $12 \mathrm{~h}$ and $24 \mathrm{~h}$ (Additional file 2: Fig. S6).

Analysis of DEGs and trends of gene expression among different samples. Between BC-0h and HS-0h (HS-0h/BC-0h), there were 24189 DEGs (24\% of all unigenes) identified, the largest number in all group comparisons. Between NC-48h and HS-48h (HS-48h/NC-48h), there were 21,513 DEGs accounting for $21.5 \%$ of all unigenes. The detailed numbers of DEGs among each pair of samples were shown in Fig. 2.

Trend analyses performed using the software STEM (Short Time-series Expression Miner) indicated that three trend analyses were designed to track changes over time after the different treatments Trend analysis I 


\section{KEGG pathway annotation}

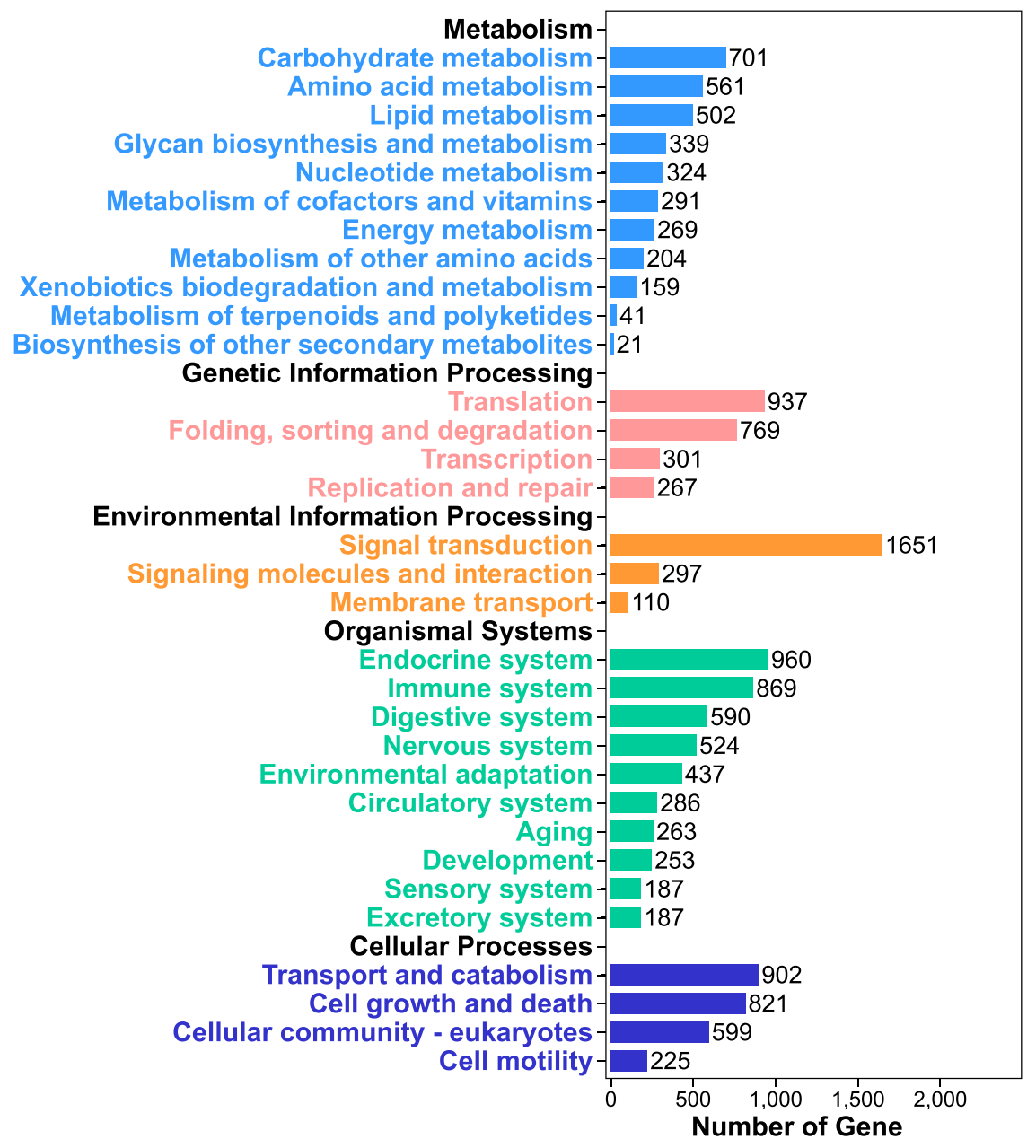

Figure 1. The Kyoto Encyclopedia of Genes and Genomes (KEGG) classification of all the assembled unigenes. A total of 10,840 unigenes had significant matches in the KEGG database and were assigned to five KEGG categories.

(BC-0h, BC-4h, BC-12h, BC-24h, BC-48h), trend analysis II (BC-0h, NC-4h, NC-12h, NC-48h), and Trend analysis III (HS-0h, HS-4h, HS-12h, HS-24h, HS-48h). A considerable number of the genes were found progressively down-regulated over time after $V$. parahaemolyticus infection. Also, with time duration after bacterium infection after hypoxia stress (trend analysis III), a total of 11,813 unigenes became down-regulated, accounting for $48 \%$ of the total DEGs $(24,246)$ (Additional file 2: Fig. S7).

Validation of the RNA-seq data by qRT-PCR. Most of the 225 immune-related genes were significantly differentially expressed after exposure to hypoxia stress and bacterial challenge (HS/NC). The validation of the DEGs revealed by RNA-Seq data with qRT-PCR analysis confirmed the expression profiles of $H$. diversicolor in response to hypoxia and bacterial infection (Additional file 1: Table S3).

The expression of each gene in the RPKM pathway in the experimental group (HS) was compared with the control group (NC) and the fold change between groups (HS/NC) was calculated to show the change of gene expression as a result of the hypoxia plus bacterial infection. In addition, the relative expression in the control group (NC) was compared with the blank group (BC) and the $\mathrm{NC} / \mathrm{BC}$ fold change was calculated to show the change as a result of infection by bacteria alone. The relative expression of 24 of the 41 genes $(P 38, R A C 1$, ASK1, MAPKAPK2, TRAF2, PPP5C, MAX, MEF2C, PRAK, bax, Apaf1, caspas3, caspas9, caspas10, caspas6, P53, $M Y D 88, N F-\kappa B, P I 3 K, E I F 4 B, E I F 4 E, F A K, I K K, A K T)$ was lower after hypoxia plus bacterial infection (HS) than with bacterial infection alone (NC) at the early stage $(4 \mathrm{~h})$. However, the expression of these genes increased at $12 \mathrm{~h}$ and $24 \mathrm{~h}$ and then decreased at $48 \mathrm{~h}$ after hypoxia plus bacterial infection (HS). After bacterial infection alone, the expression of these genes increased rapidly at $4 \mathrm{~h}$ and then declined over time when compared with blank control (BC) (Additional file 2: Fig. S8).

Analysis of qRT-PCR (Additional file 2: Fig. S9) showed that 27 of the differential expressed unigenes were consistent with the results of the transcriptome analysis with the same expression pattern. A higher expression under hypoxic stress at $12 \mathrm{~h}$ and $24 \mathrm{~h}$, and a significantly lower relative expression level at $48 \mathrm{~h}$ post infection. These genes were found to be mainly associated with signaling pathways involved in immunity, including: the 


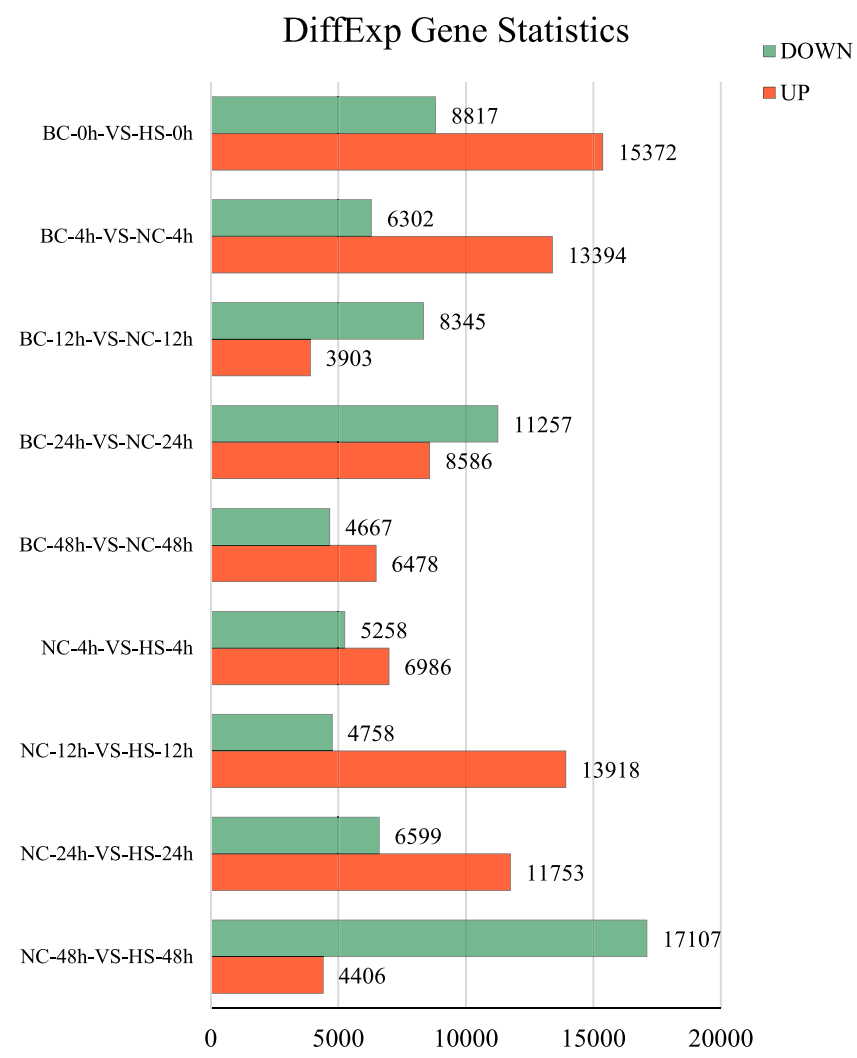

Figure 2. The number of DEGs between different groups at all sample times $(4 \mathrm{~h}, 12 \mathrm{~h}, 24 \mathrm{~h}$ and $48 \mathrm{~h}$ after injection). BC: Blank control group, normal conditions; NC: Control group, bacterial challenge under normal conditions; HS: Experimental group, hypoxia stress and bacterial challenge. There were 24189 DEGs between $\mathrm{BC}-0 \mathrm{~h}$ and HS-0h (HS-0h/BC-0h) and this number of DEGs accounted for $24 \%$ of all unigenes, the largest number of DEGs in all groups. Second, there were 21513 DEGs between NC-48h and HS-48h (HS-48h/NC$48 \mathrm{~h}$ ), accounting for $21.5 \%$ of all unigenes. FDR and $\log 2 \mathrm{FC}$ were used to screen the DEGs, and the screening condition was $\mathrm{FDR}<0.05$ and $|\log 2 \mathrm{FC}|>1$ by edgeR (An R software package for processing significant differences between paired samples).

PI3K-AKT signaling pathway ( $A K T, P I 3 K, E I F 4 B, F A K, I K K, F A S L G, I L K)$, the MAPK signaling pathway (MSK1, $M K K K 5, N L K, M K K 3, P 38)$, and the P53 signaling pathway (P53, caspas3, caspas8, caspas6).

The log2 (fold change) of the RPKM values for each group was calculated, and a heat map was used to exhibit the expression of the 41 immune-related genes. All 27 immune-related genes had the same expression pattern. The RPKM analysis of these 41 immune-related genes (Fig. 3) and the qRT-PCR analysis (Fig. 4) showed that the data from qRT-PCR were consistent with the RNA-seq results. The complete heat map of 41 immune-related genes as shown in Additional file 2: Fig. S10.

Expression of PI3K-AKT signaling pathway-related genes when the HdAKT was inhibited by dsRNA. The expression of HdAKT measured by qRT-PCR showed significantly lower at all times tested $(4 \mathrm{~h}$, $12 \mathrm{~h}, 24 \mathrm{~h}$ ) than that in the green fluorescent protein (GFP) gene group and the blank control $(p<0.05)($ Fig. 5A).

After HdAKT was inhibited by dsRNA in hemocytes, other genes in the PI3K-AKT signaling pathway showed that the expression of $A K T I P, \beta$-catenin, HdAKT, FALSG, and ILK were significantly lower at $4 \mathrm{~h}$, expression of $A K T I P, \beta$-catenin, HdPI3K, EIF4B, FAK, RHEB, HdAKT, FALSG and ILK were significantly lower at $12 \mathrm{~h}$, and expression of AKTIP, $\beta$-catenin, HdPI3K, EIF4B, FAK, IKK, RHEB, HdAKT, FALSG and ILK were significantly lower at $24 \mathrm{~h}$ compared with the GFP group and the blank control group (Fig. 5B-J).

The expressions of TSC1 and EIF4E indicated no significant effect $(P>0.05)$ in all phases after the HdAKT was inhibited by dsRNA. But in contrast, the expressions of TSC2 and $m$ TOR were significantly higher compared with that in the GFP group and the blank control group after $4 \mathrm{~h}$ of $H d A K T$ inhibition $(p<0.05)$ (Fig. 5K-N). Overall, most PI3K-AKT signaling pathway-related genes were down-regulated when the HdAKT was inhibited by dsRNA, some genes showed up-regulated mRNA expression or no significant changes $(P>0.05)$ in hemocytes. The molecular network of the PI3K-AKT signal pathway genes after HdAKT was inhibited was mapped with Cytoscape software (Fig. 6).

\section{Discussion}

Heavy mortality in hot summers and subsequent outbreak of bacterial pathogen occurred often in the farmed $H$. diversicolor ("small abalone") and threatened the sustainability of the economically important industry in China. Stress is well known to suppress immune responses, and investigations into the stress response mechanisms of $H$. diversicolor have hypothesized a link between environmental stresses (hypoxia and/or thermal stress) and 


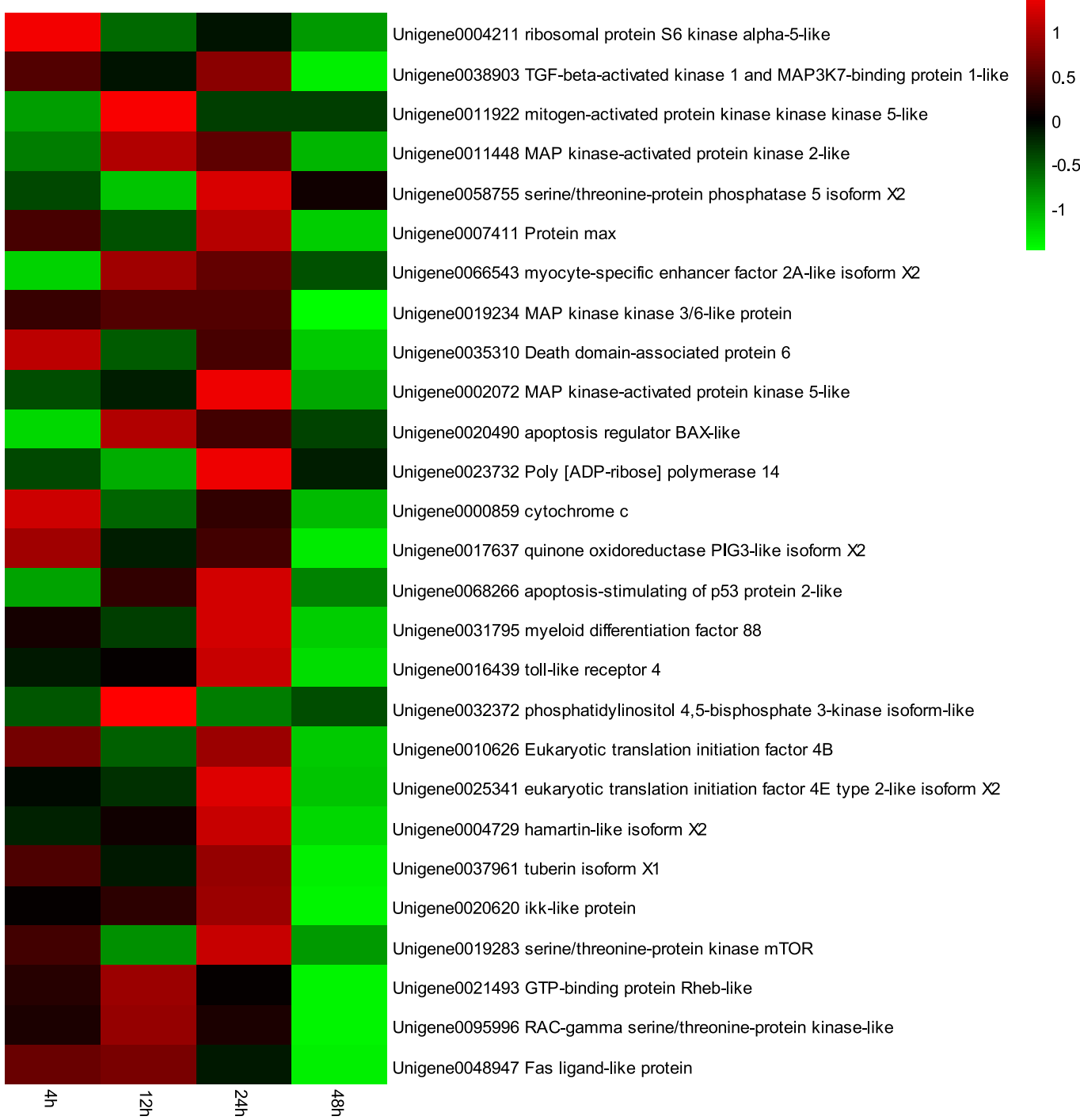

Figure 3. A heat map of the RPKM values for the relative expression of 27 immune-related genes under hypoxia stress and bacterial challenge (Experimental group, HS). The color scale at the far right of the heat map represents the RPKM relative expression value (log2 HS/NC), where red, green and black colors indicate upregulation, down-regulation and unaltered expression, respectively, relative to the NC Control group.

increased susceptibility to bacterial pathogens $s^{10,11,13,14}$. One of the vital factors that hinders the management of disease outbreaks is the inadequacy of our understanding of the molecular basis of the abalone's environmental stress response. Thus, studying the de novo transcriptomic analysis of $H$. diversicolor that has been challenged with multiple stressors is of great importance in understanding the molecular mechanisms that suppress the immune response to pathogenic bacteria.

Sequence similarity searches showed that one could assign $48.3 \%$ of the unigenes using one or more of the 4 databases used. Of these, most (97.8\%) were assigned to the 687 species in the $\mathrm{Nr}$ database, which provides a strong foundation for excavating immune-related genes and signaling pathways in the stress response. In the $\mathrm{Nr}$ database, $17 \%$ of unigenes showed homology with Pacific oysters, and 15\% of unigenes showed homology with the gastropod Elysia chlorotica. Most of the top-ranked species were marine mollusks. These results indicate that the transcript data has high reliability.

Stress levels are usually the result of many interactions among multiple environmental variables and their intensity, duration and frequency. In the present study, non-redundant genes from the $\mathrm{Nr}$ and Swissprot databases indicated a large number of DEGs of $H$. diversicolor were involved in environmental stress and/or bacterial infection. This kind of change in gene expression levels upon stress and bacterial challenge is common, and it has been observed in previous work in our lab ${ }^{10}$.

Analysis of GO and KEGG annotation. As expected, the annotation of the unigenes by Blast2GO identified genes involved in a variety of cellular activities. $22.6 \%$ of the unigenes were allocated to functional categories. This allows us to quickly find the target genes and locate the relevant signaling pathways. 


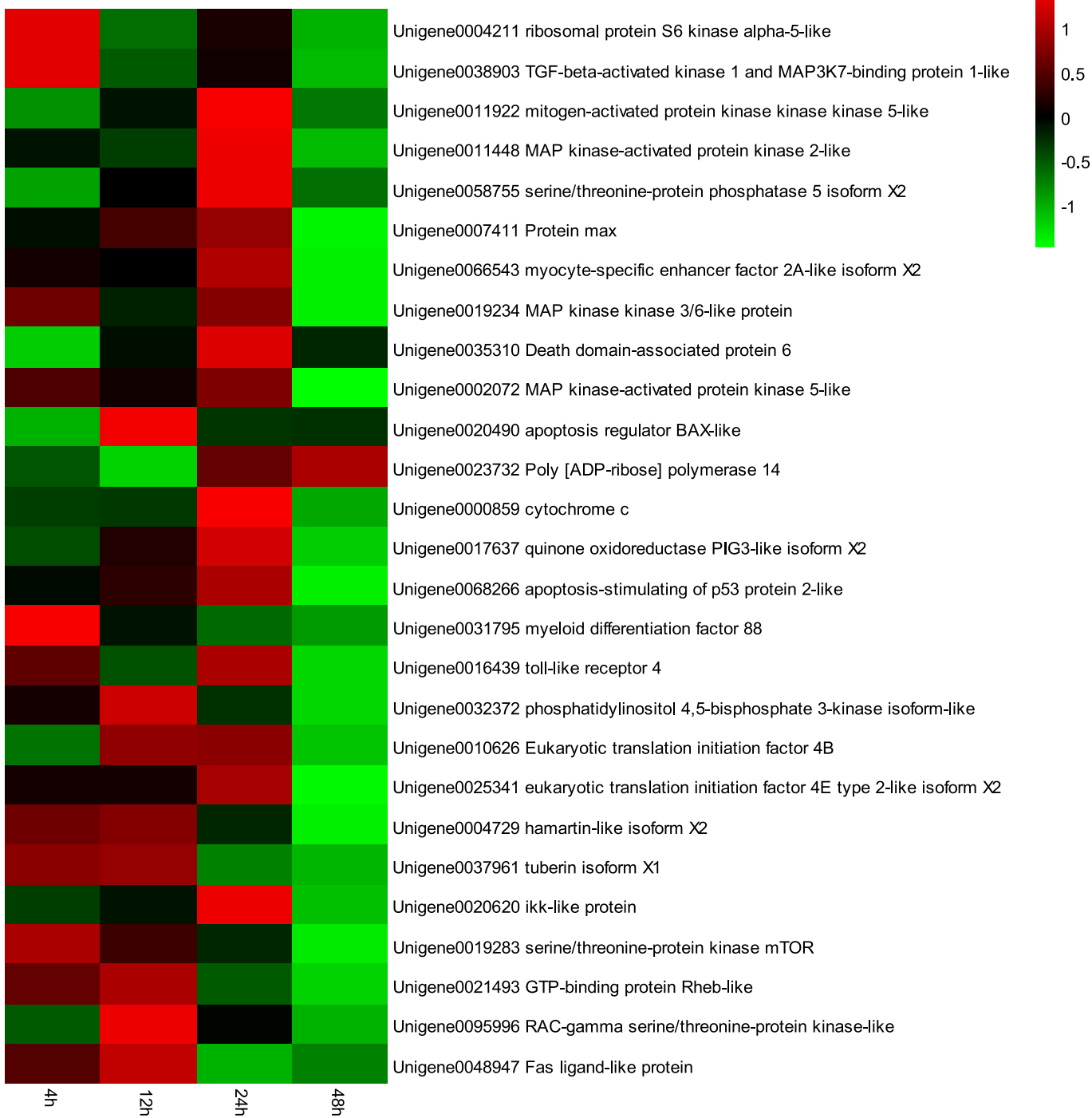

Figure 4. A heat map of the relative expression using qRT-PCR of 27 immune-related genes at hypoxia stress and bacterial challenge (Experimental group, HS). The color scale at the far right of the heat map represents the relative mRNA expression level (log2 HS/NC), where red, green and black colors indicate up-regulation, downregulation and unaltered expression, respectively, relative to the NC Control group.

Annotation of unigenes in the KEGG pathway database showed that many pathways may be related to the immune defense response of $H$. diversicolor. Multiple immune-related and apoptosis-related pathways were found, including the MAPK signaling pathway, the P38 pathway, the NF- $\kappa$ B signaling pathway, the HIF signaling pathway under hypoxia stress, heat shock genes that play an important role in high temperature stress, and the PI3K-AKT pathway with immunosuppression under stress. The discovery and identification of these immune-related pathways has laid a foundation for the study of the mechanism of immune function genes under various environmental stressors.

Differentially expressed genes and qRT-PCR validation. The number of DEGs in each group varies, with different expression patterns among the groups. Not surprisingly, the largest number of DEGs was between the blank control and the hypoxia stressed group, immediately after the injection. $24 \%$ of all unigenes were differentially expressed in this comparison, where, $59 \%$ of these genes were annotated indicates that the DEGs can provide very useful information about the responses to bacterial challenge and environmental stress. In addition, the results of qRT-PCR analyses of those genes showed that the expression patterns over time were in agreement with the Illuminia RNA-Seq analyses.

Discovery of immune-related genes. Of the large number of unigenes identified through RNAseq, a relatively small number were related to immune function. As described in the introduction, the PI3K-AKT signal transduction pathway is an evolutionarily conserved innate immune pathway, which plays a critical role to resist 


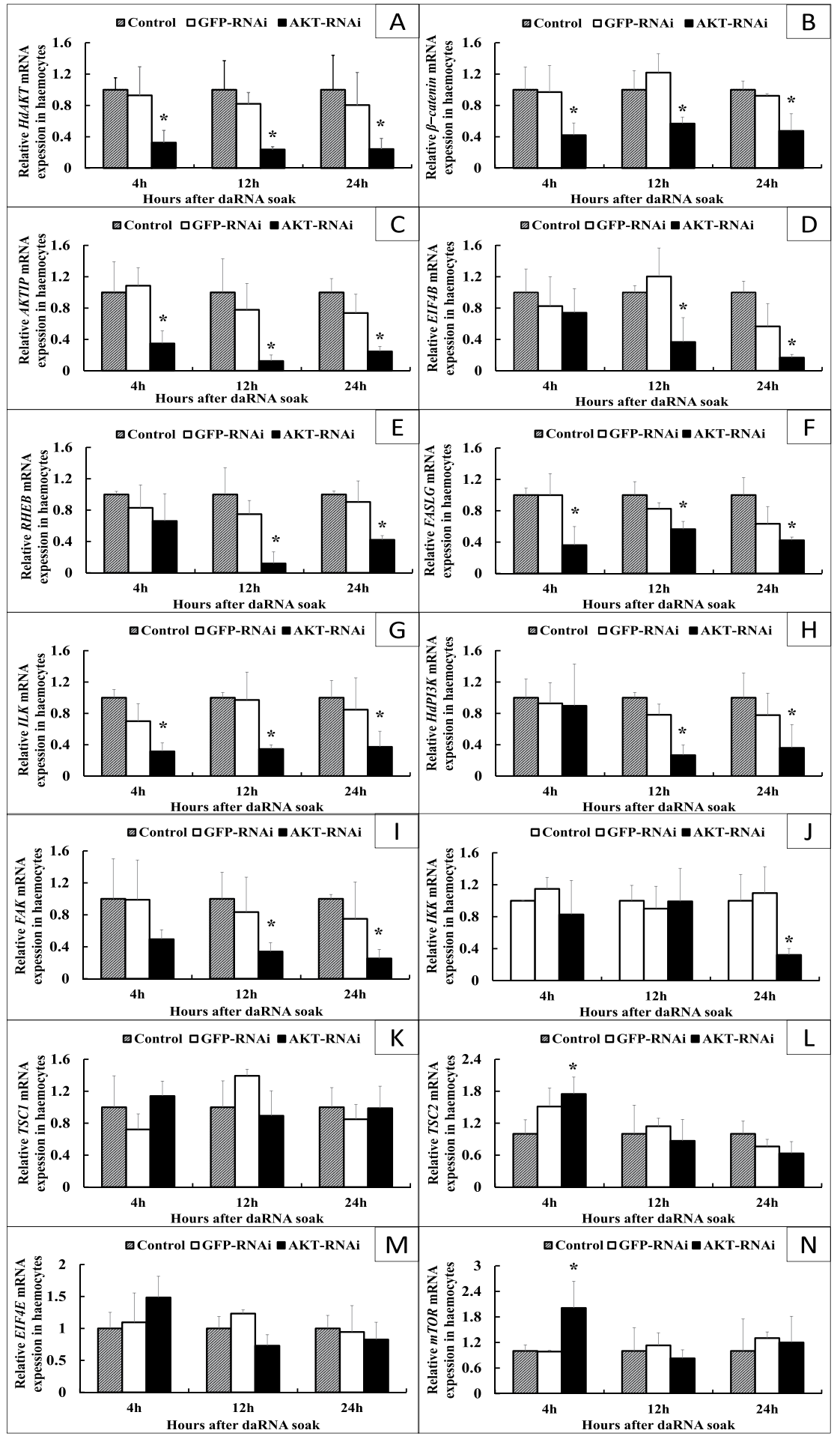

Figure 5. Expression analysis of the PI3K-AKT signaling pathway related genes when the HdAKT was inhibited by dsRNA in haemocytes. X axis: Sample times - hours after HdAKT was inhibited by dsRNA ( $4 \mathrm{~h}$, $12 \mathrm{~h}$ and $24 \mathrm{~h}$ ). Y axis: mRNA expression level of the PI3K-AKT signaling pathway related genes. The significant difference between the AKT-RNAi group and the control group is indicated by a $(*)$ at $p<0.05$. $\beta$-actin served as reference gene. Control: blank control group. GFP-RNAi: group in which green fluorescent protein (GFP) gene was inhibited by dsRNA. AKT-RNAi: group in which HdAKT was inhibited by dsRNA.

external pathogens and stressors from invertebrates to vertebrates ${ }^{49-53}$. One report on Crassostrea hongkongen$s i s^{54}$ suggested that AKT1 participates vigorously in the immune response against microbial pathogens and heat shock stresses. Additionally, PI3K signaling pathway was reported to involve in regulating glycogen metabolism in Pinctada fucata ${ }^{55}$. Furthermore, transcriptome analysis showed that PI3K-AKT signaling pathway involved 


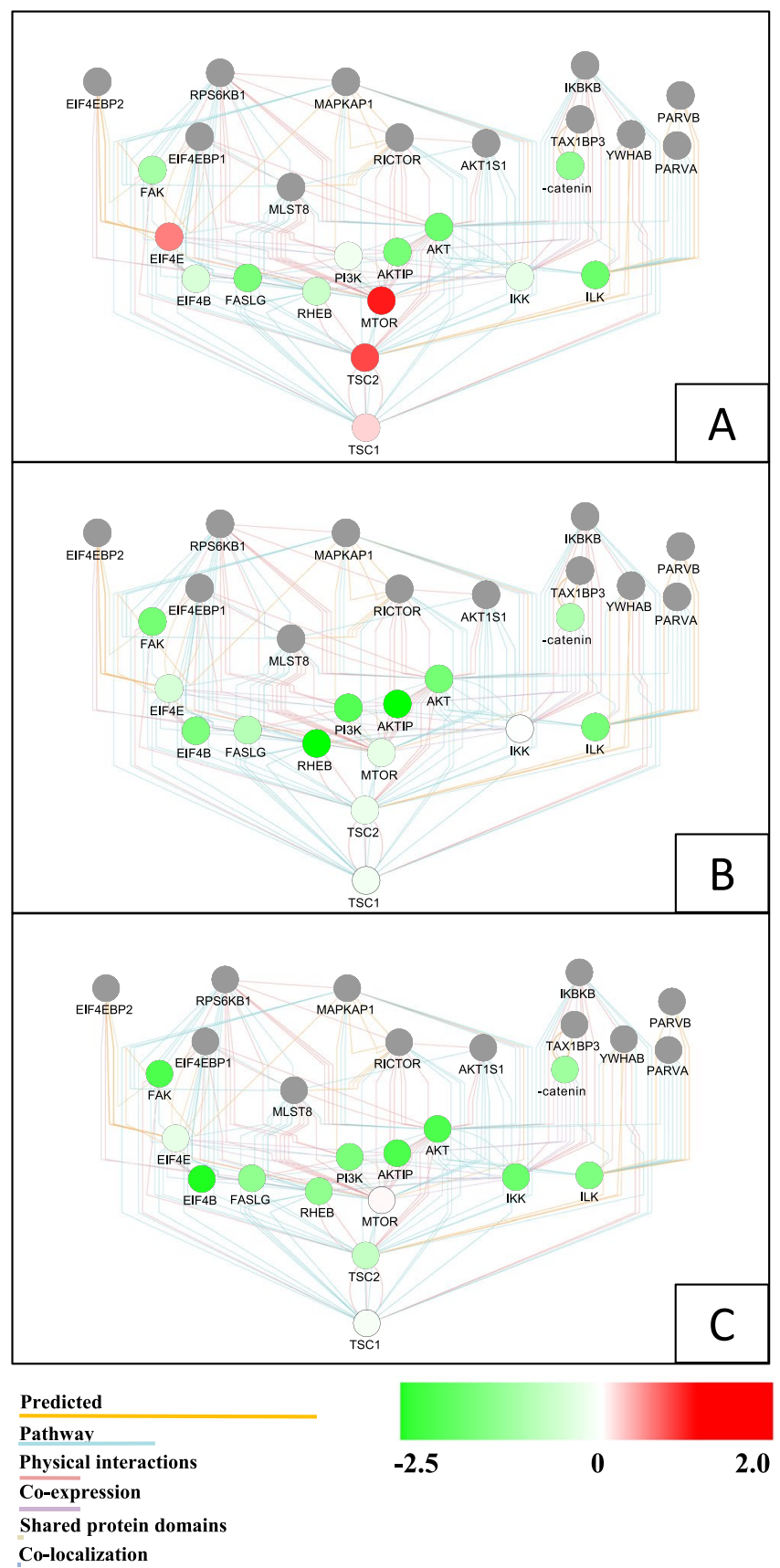

Figure 6. The networks of the PI3K-AKT signaling pathway related genes, representing the level of regulation of PI3K-AKT signaling pathway related genes compared with the blank control group in haemocytes, at various times when the HdAKT was inhibited by dsRNA. (A) after $4 \mathrm{~h},(\mathbf{B})$ after $12 \mathrm{~h},(\mathbf{C})$ after $24 \mathrm{~h}$. Red, green and white colors indicate up-regulation, down-regulation and unaltered expression, respectively. The genes not subject to research are marked in gray.

in immune defense response to pathogenic infection and environmental stress in Mytilus coruscus ${ }^{56}$, Onchidium struma $^{57}$ and Meretrix petechialis ${ }^{58}$. In addition, the PI3K-AKT signaling pathway related to TLR4-mediated immune defense ${ }^{59,60}$, that similar with the abalone $H$. diversicolor after the V. parahaemolyticus stimulation ${ }^{14}$.

The expression of $A K T, P I 3 K$, EIF4B, FAK, IKK, FALSG, ILK and other related genes in the PI3K-AKT signal pathway under hypoxia stress decreased compared to those under normal conditions as the time after bacterial infection increased, and at $48 \mathrm{~h}$ after infection no differences were found between the hypoxia stress group and control group. However, the expression of PI3K-AKT signaling pathway-related genes increased rapidly at $4 \mathrm{~h}$ after bacterial infection and then declined over time compared with blank control (NC/BC), suggesting that this pathway is involved in the rapid immune response to infection. Also, hypoxia stress delays this rapid immune response, indicating that the PI3K-AKT signaling pathway may be involved in hypoxia-induced immunosuppression of $H$. diversicolor under hypoxia stress when infected with $V$. parahaemolyticus. Therefore, further research 
on the PI3K-AKT signaling pathway under the combined environmental stresses and pathogen invasion would be fruitful.

In vertebrates, members of the MAPK signaling pathway belong to the family of protein kinases that catalyze the phosphorylation of proteins and form intricate regulatory networks that regulate gene expression and play a critical functional role in cell proliferation, apoptosis, immune defense and humoral immunity ${ }^{61}$. As important members of the serine/threonine protein kinase family, a cascade reaction of kinases activates nuclear transcription factors in stages through MAPK, MKK and MKKK. This cascade reaction of kinases plays an important role in immune defense and developmental regulation.

The MAPK pathway includes four sub-pathways: an extra-cellular signal-regulated kinase (ERK) signaling pathway, a Jun N-terminal kinase (JNK) pathway, the P38 signaling pathway and an extracellular signal-regulated kinase signaling pathway. These four pathways are interlinked and interact with each other to form an extensive information transmission network that constitutes the regulation mechanism for immune defense and developmental and reproductive function. The MAPK signaling pathway is an important immune-related pathway in vertebrates, and also is involved in immune defense against pathogenic bacteria in mollusks in Mytilus galloprovincialis ${ }^{62}$, Crassostrea ariakensis ${ }^{63}$ and Haliotis tuberculate ${ }^{64}$. Previous transcriptome analysis showed that MAPK signaling pathway involved in immune response, and the expression of these genes varied significantly during bacterial infec-

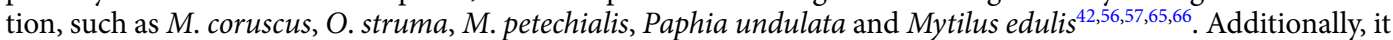
is also involved in immune defense under environmental stress in O. struma ${ }^{53}$. Relatively, little research on this pathway in mollusks has been reported, and the available reports mostly focused onto bacterial immune defense.

In this study, a total of 62 MAPK signaling pathway related genes were obtained, including key factors in multiple pathways: $M S K 1, M K K K 5, N L K, M K K 3, P 38$ etc. Our transcriptome analysis of these related genes in the MAPK signaling pathway showed that the expression levels of these genes appeared to decline to varying degrees after $V$. parahaemolyticus infection under hypoxia stress especially at $4 \mathrm{~h}$ and $48 \mathrm{~h}$ (HS). But the expression levels of these genes increase rapidly at $4 \mathrm{~h}$ after bacterial infection and then decline over time compared with blank control. As for the P13K-AKT pathway, this suggests that the MAPK signaling pathway may be involved in the rapid immune response after $V$. parahaemolyticus infection and hypoxia-induced immunosuppression of $H$. diversicolor. This provides a basis for studying the immune regulation mechanism of the MAPK signaling pathway in more complex environmental stress and pathogen infection experiments.

As a highly conserved pathway from insects to mammals, the nuclear factor- $\kappa B(N F-\kappa B)$, which plays central roles in many important physiological and pathological processes, has been studied extensively in the innate immune system ${ }^{67,68}$. NF- $\kappa B$ is present in most vertebrates and invertebrates; and it has been described as a nuclear factor which can specifically bind to the $\kappa B$ sequence of immunoglobulin $\kappa$ enhancer ${ }^{69}$. More recently, it has been identified in almost all animal cell types and has become well known as a transcription factor involved in many biological processes such as development, immune defense, inflammatory responses, apoptosis, homeostatic mechanisms and cellular differentiation ${ }^{70}$.

Previous transcriptome analysis have shown that NF- $\kappa B$ signaling pathway plays a pivotal role in immune response, and the expression of these genes varied significantly during bacterial infection in O. struma, Concholepas concholepas and M. edulis ${ }^{57,66,71}$. Further, in a recent study of the transcriptome KEGG analyses suggested that the NF- $\kappa B$ signaling pathway were actively expressed in the organ and might serve as a host defense modulator against exogenous infections such as bacteria and other pathogens in Saxidomus purpuratus ${ }^{72}$. Meanwhile, after recognition of the invasive pathogens, NF- $\kappa$ B signaling pathway are triggered, and this pathway is considered a crucial component in innate immunity, as it plays an important role in the innate defense against common pathogens in P. undulat ${ }^{65}$. Our previous results have shown that the expressions of NF- $\kappa B$ signaling pathway-related genes were up-regulated significantly under hypoxia stress, thermal stress and both stressors together, as well as bacterial infection ${ }^{10}$. This indicates that the NF- $\kappa B$ signaling pathway is involved in both the innate immune response and in immune regulation under multiple environmental stressors.

In this study, a total of twenty NF- $\kappa B$ signaling pathway related genes were obtained, which provides a foundation for subsequent screening of key genes for immunity. In the transcriptome trend analysis, the expression of $N F-\kappa B$ showed a downward trend. But after bacterial infection alone (NC) there was a rapid increase of expression at $4 \mathrm{~h}$. As hypoxia stress delays the rapid immune response after bacterial infection, these results indicate that the NF- $\kappa B$ signaling pathway is involved in immunosuppression of $H$. diversicolor under the combined stress of hypoxia stress and vibrio infection.

The P53 signaling pathway plays a key role in the process of apoptosis regulation and is closely related to the biological regulation of cell growth, differentiation and immunity in vertebrates ${ }^{73}$. Our transcriptome analysis revealed twenty-four P53 signaling pathway-related genes that are closely related to apoptosis and immune function in $H$. diversicolor. We have also obtained $18 \mathrm{HIF}$ signaling pathway-related genes that are important for the response to a hypoxic environment. In addition, 4 heat shock response-related genes were obtained; these genes are of great significance to cope with a high temperature stimulus.

The 41 immune-related unigenes that were selected on the basis of their roles of genes in various immune pathways as immune-related genes, mainly belonged to the PI3K-AKT, MAPK, NF- $\kappa$ B and P53 signaling pathways. These unigenes were verified by qRT-PCR and the expression patterns of 27 of these unigenes were consistent with the transcriptome analysis.

Based on the analysis of the heat-map and the gene expression patterns, 24 genes in haemocytes that respond to bacterial infection (NC) and hypoxia plus bacterial infection (HS) can be generally classified as showing the following response pattern: The genes increase rapidly at $4 \mathrm{~h}$ after bacterial infection alone, and then decline over time compared with blank control (BC), suggesting that they are involved in the rapid immune response to bacterial infection. But after hypoxia plus bacterial infection (HS) they were expressed at a low level compared with bacterial infection (NC) at $4 \mathrm{~h}$, and their expression increased at $12 \mathrm{~h}$ and $24 \mathrm{~h}$, then decreased at $48 \mathrm{~h}$. The expression of these genes is thus correlated with the delay in the response to bacterial infection under hypoxia 
stress. To some extent, these immune-related genes may be involved in the process of hypoxia-induced immunosuppression of $H$. diversicolor after $V$. parahaemolyticus infection with hypoxia stress. Many of these genes are associated with immune signaling pathways, such as the P13K-AKT pathway.

The regulation mechanism of PI3K-AKT signaling pathway when the HdAKT was inhibited by dsRNA. The technology of gene silencing by dsRNA (RNAi) has been applied recently to marine mollusks, such as a freshwater snail Biomphalaria glabrata for studying the function of genes in host-parasite interactions with trematode parasites ${ }^{74}$. The genes activated by AKT indicated the inflammatory or immune response, a cell survival response or cellular proliferation. To study the mechanism of AKT in the PI3K-AKT signaling pathway, the HdAKT gene was inhibited by dsRNA in the blood cells of $H$. diversicolor.

qRT-PCR showed that the expression of HdAKT in the experimental groups inhibited by dsRNA was significantly lower than in the GFP gene group and the blank control, and HdPI3K and pathway-related genes were significantly down-regulated in the experimental group of hemocytes. This shows that HdAKT plays a positive role in the regulation of the PI3K-AKT signaling pathway. The expression levels of other genes in the P13K-AKT pathway, except EIF4E, TSC1, TSC2 and $m T O R$, were lower than that in the control group after HdAKT was inhibited for $4 \mathrm{~h}$, indicating $H d A K T$ plays a key role in the regulation of PI3K-AKT signaling pathway.

The unchanged expressions of EIF4E, TSC1, TSC2 and $m$ TOR genes at $4 \mathrm{~h}$ and longer after HdAKT was inhibited suggested that these genes are located upstream of the PI3K-AKT signaling pathway, and may uni-directionally regulate $H d A K T$. The interactions between PI3K-AKT signaling pathway-related genes were also visualized by Cytoscape after the inhibition of HdAKT. From the molecular network, it is reflected that inhibition of $H d A K T$ deepens as the inhibition time lengthens, and these genes gradually appear to be inhibited. As a supplementary experiment, the inhibition of HdAKT helped to understand the mechanism of PI3K-AKT signaling. These results provide evidence of the PI3K-AKT signaling linkages between these genes in abalone.

\section{Conclusions}

Transcriptome profile of $H$. diversicolor hemocytes revealed that the expression of immune defense genes in the PI3K-AKT, MAPK, NF- $\kappa$ B and P53 signaling pathways under hypoxia stress decreased relative to their expression under normal conditions, as the time after bacterial infection increased. But the expression levels of these genes increased rapidly at $4 \mathrm{~h}$ after bacterial infection and then declined over time compared with blank control. The results indicated that the hypoxia stress delays this rapid immune response and the immune defense genes may be involved in hypoxia-induced immunosuppression of $H$. diversicolor. The silencing of $H d A K T$ downregulated the expression of other genes in the P13K-AKT pathway, except EIF4E, TSC1, TSC2 and mTOR. Furthermore, the gene interaction network of the PI3K-AKT signal pathway was successfully constructed after HdAKT was inhibited, by Cytoscape software. These results contribute substantially to exploring the changes of the immune system in marine mollusks under environment stress conditions.

\section{Materials and Methods}

Animals and sample preparation. Adult small abalone at body length of $6.00 \pm 0.50 \mathrm{~cm}$ and body weight of $16.45 \pm 2.50 \mathrm{~g}$ were purchased from Peiyang abalone farm (Xiamen, Fujian Province) in August 2015. Following the methods in our previous publication ${ }^{10}$, these abalone were kept in recirculating systems with sand-filtered seawater at a consistent temperature $\left(25^{\circ} \mathrm{C}\right)$ and dissolved oxygen (DO) $(6.2 \mathrm{mg} / \mathrm{L})$, and fed with Laminaria japonica once a day. During experimental period, the temperature and the level of DO of the seawater were monitored continuously and maintained using a temperature and dissolved oxygen control system (Xiamen Water Bay Automation Technology Co., Ltd, Xiamen, China). Before the challenge experiments, abalones were acclimated in the system for 10 days.

Challenge experimental designation. After acclimation, abalones were randomly divided into three groups for the following treatments: (1) Normal control group (NC) subjected to bacterial challenge under normal conditions. After these abalone had been cultured under the normal conditions $\left(\mathrm{T}=25^{\circ} \mathrm{C}\right.$ and $\left.\mathrm{DO}=6.2 \mathrm{mg} / \mathrm{L}\right)$ for $96 \mathrm{~h}$, all individuals were injected with $50 \mu \mathrm{L}$ live $V$. parahaemolyticus in $0.9 \% \mathrm{NaCl}\left(1.1 \times 10^{8} \mathrm{cfu} / \mathrm{mL}\right)$ into the foot muscle. (2) Blank control group (BC) with no bacterial challenge under normal conditions. After these abalone had been cultured under normal conditions for $96 \mathrm{~h}$, they were injected with $50 \mu \mathrm{L}$ of $0.9 \% \mathrm{NaCl}$. (3) An experimental group subjected to bacterial challenge after hypoxia stress (HS). These abalone were cultured at $25^{\circ} \mathrm{C}$ and DO of $2 \mathrm{mg} / \mathrm{L}$ for $96 \mathrm{~h}$, and then injected with $50-\mu \mathrm{L}$ of live $V$. parahaemolyticus in $0.9 \% \mathrm{NaCl}\left(1.1 \times 10^{8}\right.$ $\mathrm{cfu} / \mathrm{mL}$ ) into the foot muscle. After the treatments of injection stated above, the abalone were returned to their original tanks containing seawater at $\mathrm{T}=25^{\circ} \mathrm{C}$ and $\mathrm{DO}=6.2 \mathrm{mg} / \mathrm{L}$. At $0,4,12,24$ and $48 \mathrm{~h}$ post-injection, the abalones from each treatment group were sampled and tissues were collected as follows: Hemolymph was drew through cutting off the foot using a syringe with 21 -gauge needle, and centrifuged at $2000 \times \mathrm{g}$ at $4{ }^{\circ} \mathrm{C}$ for $10 \mathrm{~min}$ to separate hemocytes $(\mathrm{He})$. The hemocytes were immediately stored in liquid nitrogen until used for RNA isolation (Fig. 7). As $0 \mathrm{~h}$ was sampled immediately after injection, $\mathrm{BC}-\mathrm{Oh}$ is equivalent to $\mathrm{NC}-0 \mathrm{~h}$, and only the $\mathrm{BC}$ group was sampled. In all of the treatments, at least six abalone were sampled at each time.

Double-stranded RNA (dsRNA) generation and exposure assay. A fragment of $H d A K T$ (whose complete cDNA has been cloned with GenBank accession No. KX056492) and green fluorescent protein (GFP) gene from the pEGFP-N1 vector were amplified by PCR using gene-specific primers (Additional file 1: Table S4). The primers of the $H d A K T$ fragment include sense primers and antisense primers of both $H d A K T$ and $g f p$ genes for transcribing single-stranded RNA). The PCR products were purified, sequenced and transcribed into single-stranded RNA (ssRNA) using the T7 phage RNA polymerases (Promega, Madison, WI, USA), and then 


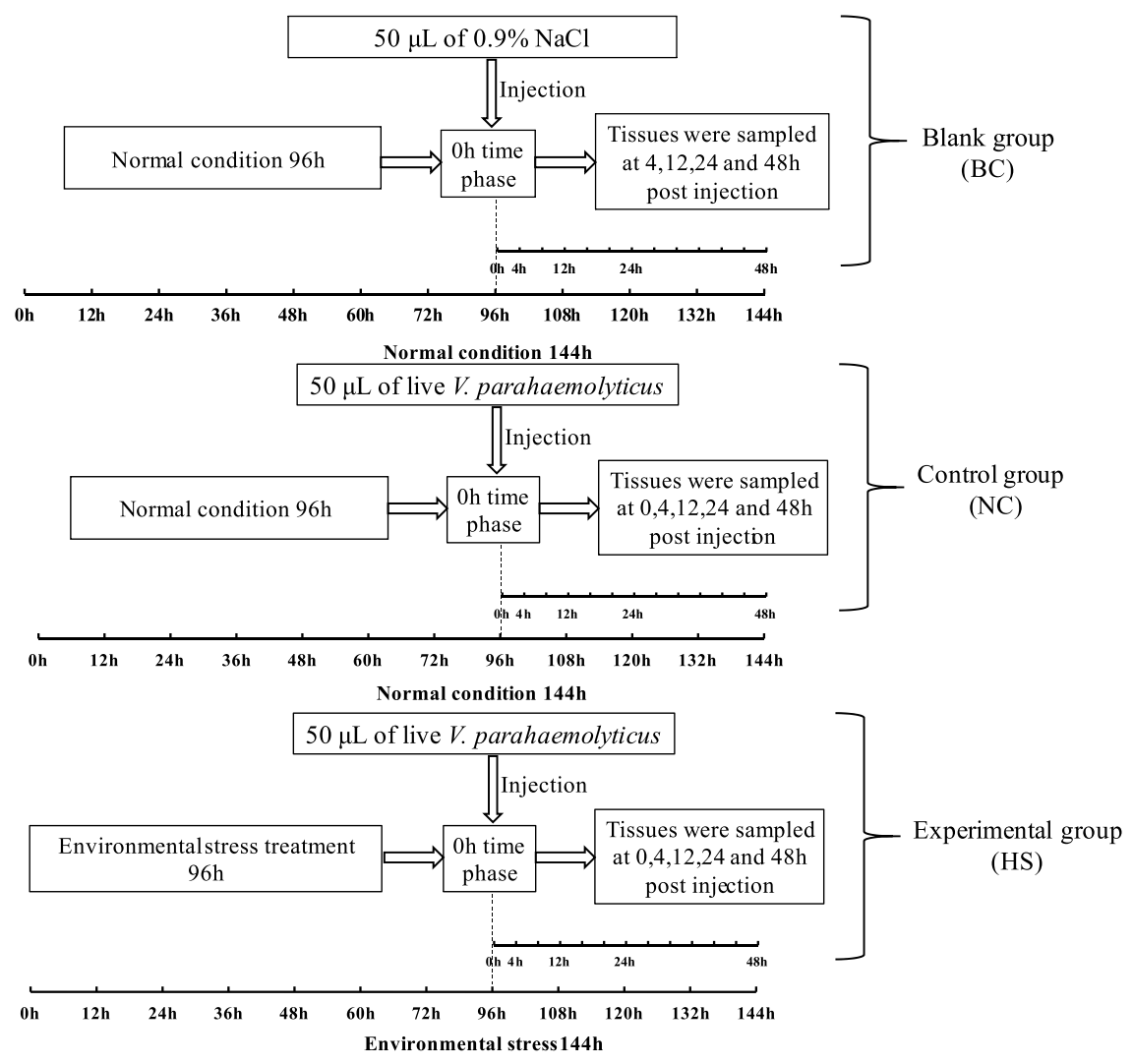

Figure 7. Schematics of the experimental set-up. The Blank group (BC) were kept under normal conditions and injected with $50 \mu \mathrm{L} 0.9 \% \mathrm{NaCl}$ at $96 \mathrm{~h}$. The Experimental group (HS) were challenged by injection with $50 \mu \mathrm{L}$ live $V$. parahaemolyticus (isolated from diseased abalone) in $0.9 \% \mathrm{NaCl}\left(1.1 \times 10^{8} \mathrm{cfu} / \mathrm{mL}\right)$. These abalone were exposed to the stress of hypoxia during the whole experiment. The Control group (NC) was kept under normal conditions but challenged by injection of $50 \mu \mathrm{L}$ live $V$.parahaemolyticus (isolated from diseased abalone) in $0.9 \% \mathrm{NaCl}\left(1.1 \times 10^{8} \mathrm{cfu} / \mathrm{mL}\right)$ as for $\mathrm{HS}$.

the DNA template was degraded by DNase I (Promega) at a ratio of $1 \mathrm{U} / \mu \mathrm{g}$. The sense ssRNA and antisense ssRNA were mixed and annealed at $75^{\circ} \mathrm{C}$ for $15 \mathrm{~min}, 65^{\circ} \mathrm{C}$ for $15 \mathrm{~min}$, and then cool down to room temperature $\left(\mathrm{T}=25^{\circ} \mathrm{C}\right)$ at the rate of $0.1{ }^{\circ} \mathrm{C} / \mathrm{s}$. The quality of dsRNA and size shift was assessed by agarose ( $\left.1 \%\right)$ gel electrophoresis and the concentration of the dsRNA was assessed by spectrophotometry (NanoDrop ND-1000, Thermo Fisher Scientific, Wilmington, DE, USA).

To achieve the RNA interference (RNAi), the exogenous dsRNA at a final concentration of $5 \mu \mathrm{g} / \mathrm{ml}$ was added directly to the hemocyte culture medium without any vehicle. The HdAKT dsRNA was applied to hemocytes in the experimental group, the GFP dsRNA was applied to hemocytes in the control group (GFP group), and medium without RNAi treatment was paralleled as blank control. After mixing and incubation at $27^{\circ} \mathrm{C}$ for 6,12 and $24 \mathrm{~h}$, the hemocytes were collected by centrifugation $2000 \times \mathrm{g}$ and stored in liquid nitrogen until they were processed to isolate the RNA and detect the mRNA expression by qRT-PCR. Six replicates from each treatment group were produced by using different individual abalones.

Isolation of total RNA and RNA-sequencing. Total RNA was extracted from all the hemocyte samples using the E.Z.N.A. ${ }^{\circledR}$ Total RNA Kit II (Omega Bio-tek, Inc.Norcross, GA, USA) following the manufacturer's instructions. The mRNA quality was assessed by agarose (1\%) gel electrophoresis and quantified by spectrophotometry (NanoDrop ND-1000). RNA sequencing was then performed by Gene Denovo Biotechnology Co. (Guangzhou, China). As input material, 3- $\mu$ g RNA per sample was used for sequencing libraries and the RNA was enriched by Oligo (dT) beads. Afterwards, the enriched mRNA was cut into short fragments and reverse transcribed into fragmented cDNA with random primers. Then, the fragments of cDNA were purified with a QiaQuick PCR extraction kit (QIAGEN China Co., Ltd., Shanghai, China), end repaired, poly(A) primer was added, and the DNA ligated to Illumina sequencing adapters. Finally, the ligation products were selected by agarose gel electrophoresis and amplified by PCR. The cDNA libraries of all samples were sequenced using the Illumina HiSeq 2000 platform (Illumina, San Diego, CA, USA) following the manufacturer's recommendations, and paired-end reads were generated.

For qRT-PCR, the total mRNA was reverse transcribed into cDNA with $1-\mu \mathrm{g}$ total RNA and $2-\mu \mathrm{L}$ of $10-\mu \mathrm{M}$ random primers by M-MLV reverse transcriptase (Promega -Beijing Biotech Co., Ltd., Beijing, China). The cDNA was diluted 100 -fold and stored at $-20^{\circ} \mathrm{C}$, ready for qRT-PCR. 
De novo assembly, functional annotation, and identification of DEGs. Trinity software was used to assemble the clean reads as described for De Novo transcriptome assembly without a reference genome, from which adaptor sequences, ambiguous ' $N$ ' nucleotides (with a ratio of ' $N$ ' more than $10 \%$ ) and low quality sequences (with quality score $<5$ ) were removed ${ }^{75}$. The raw sequence data of fourteen libraries have been deposited into the NCBI Sequence Read Archive (SRA) under the accession number SRP166258. For annotation analysis, non-redundant sequences were subjected to public databases, including NCBI (http://www.ncbi.nlm. nih.gov/), non-redundant protein $(\mathrm{Nr})$ and non-redundant nucleotide (Nt), Swiss-Prot (http://www.ebi.ac.uk/ uniprot/), Gene Ontology (GO) (http://www.geneontology.org/), Clusters of Orthologous Groups (COG) (http:// www.ncbi.nlm.nih.gov/COG/) and Kyoto Encyclopedia of Genes and Genomes (KEGG) (http://www.genome.jp/ $\mathrm{kegg} /$ ). When the results of different databases were conflicted, a priority order of alignments from $\mathrm{Nr}$, Nt, KEGG, Swiss-Prot, GO to the COG databases was followed.

All clean sequencing reads of the fourteen libraries were mapped to the transcriptome assembly using the software Bowtie2 with the default setting. Gene expression levels of unigenes were based on read counts obtained by RNA Sequence Expected Maximization, and these read counts were normalized using the Reads per Kilobase per Million mapped transformation ${ }^{76,77}$. However, differentially expressed genes (DEGs) were further analyzed by the "Identifying edge" R package (www.r-project.org) to assign them to genes in cDNA libraries of H. diversicolor. In the edge $\mathrm{R}$ statistics, files (FDR $<0.05$ and $|\log 2 \mathrm{FC}|>1$ ) were set as the threshold for significantly differential expression to identify DEGs in various libraries.

qRT-PCR verification. To further investigate the roles of genes in immune pathways, 41 representative genes were screened and selected to validate the RNA-Seq data by qRT-PCR. Gene-specific primers of these 41 genes (Additional file 1: Table S5) were designed to amplify products of 200-300 bp of the cDNA. A $10 \times$ SYBR Green Master mix (Promega) was used for qRT-PCR according to the manufacturer's protocol, and the $\beta$-actin gene (Accession No. AY436644) was selected as the housekeeping gene due to its stable expression in abalones, ${ }^{8,11,12}$.

qRT-PCR was carried out in a Light Cycler 480 Roche Real-time Thermal Cycler (Roche, Switzerland) with a $20-\mu \mathrm{L}$ reaction volume containing $9 \mu \mathrm{L}$ of 1:100 diluted original $\mathrm{CDNA}, 10 \mu \mathrm{L}$ of $10 \times$ SYBR Green Master Mix (Promega), and $0.5 \mu \mathrm{L}$ of each primer $(10 \mu \mathrm{M})$. The cycling conditions for the PCR reaction were as follows: $1 \mathrm{~min}$ at $95^{\circ} \mathrm{C}$, followed by 40 cycles at $95^{\circ} \mathrm{C}$ for $15 \mathrm{~s}, 60^{\circ} \mathrm{C}$ for $1 \mathrm{~min}$. Melting curves were also plotted $\left(60^{\circ} \mathrm{C}-90^{\circ} \mathrm{C}\right)$ to make sure that a single PCR product was amplified for each pair of primers. The comparative CT method ( $\Delta \mathrm{CT}=\mathrm{CT}$ of target gene minus $\mathrm{CT}$ of $\beta$-actin gene and $\Delta \Delta \mathrm{CT}=\Delta \mathrm{CT}$ of any sample minus calibrator sample) was used to calculate the relative expression level of all these genes. The t-test by IBM SPSS Statistics 20 was used to determine the difference in the mean values among the treatments, with a significance level of $p<0.05$. A heat map was created using the R Programming Language (version 3.4.0) to visualize the gene expression data. The gene networks of signaling pathway related genes were constructed using the Gene MANIA Cytoscape app (Cytoscape 3.4.0).

Received: 14 June 2019; Accepted: 3 December 2019;

Published online: 24 December 2019

\section{References}

1. Wang, K., Ren, H., Xu, D., Cai, L. \& Yang, M. Identification of the up-regulated expression genes in hemocytes of variously colored abalone (Haliotis diversicolor Reeve, 1846) challenged with bacteria. Developmental \& Comparative Immunology 32, 1326-1347 (2008).

2. Vaquer, Sunyer., R. \& Duarte., C. M. Temperature effects on oxygen thresholds for hypoxia in marine benthic organisms. Global Change Biology 17, 1788-1797 (2011).

3. Liu, P. C., Chen, Y. C., Huang, C. Y. \& Lee, K. K. Virulence of Vibrio parahaemolyticus isolated from cultured small abalone, Haliotis diversicolor supertexta, with withering syndrome. Letters in Applied Microbiology 31, 433-437 (2000).

4. Cheng, W., Hsiao, I.-S. \& Chen, J.-C. Effect of ammonia on the immune response of Taiwan abalone Haliotis diversicolor supertexta and its susceptibility to Vibrio parahaemolyticus. Fish \& Shellfish Immunology 17, 193-202 (2004).

5. Wang, B. et al. Molecular cloning and characterization of macrophage migration inhibitory factor from small abalone Haliotis diversicolor supertexta. Fish \& Shellfish Immunology 27, 57-64 (2009).

6. Zhang, K. et al. Cloning, characterization and TBT exposure response of CuZn superoxide dismutase from Haliotis diversicolor supertexta. Molecular Biology Reports 36, 583-594 (2009).

7. Wang, G. et al. Molecular cloning and responsive expression of macrophage expressed gene from small abalone Haliotis diversicolor supertexta. Fish \& Shellfish Immunology 24, 346-359 (2008).

8. Li, N. et al. Insulin-like growth factor binding protein 7, a member of insulin-like growth factor signal pathway, involved in immune response of small abalone Haliotis diversicolor. Fish \& Shellfish Immunology 33, 229-242 (2012).

9. Ge, H. et al. Molecular cloning and expression of interleukin-1 receptor-associated kinase 4, an important mediator of Toll-like receptor signal pathway, from small abalone Haliotis diversicolor. Fish \& Shellfish Immunology 30, 1138-1146 (2011).

10. Zhang, X. et al. Identification and expression analysis of immune-related genes linked to Rel/NF- $\kappa \mathrm{B}$ signaling pathway under stresses and bacterial challenge from the small abalone Haliotis diversicolor. Fish \& Shellfish Immunology 41, 200-208 (2014).

11. Huang, Y. et al. Molecular cloning, characterization and expression analysis of three heat shock responsive genes from Haliotis diversicolor. Fish \& Shellfish Immunology 36, 590-599 (2014).

12. Ge, H. et al. Characterization of interleukin-1 receptor-associated kinase 1 binding protein 1 gene in small abalone Haliotis diversicolor. Gene 506, 417-422 (2012).

13. Cai, X. et al. Cloning, characterization, hypoxia and heat shock response of hypoxia inducible factor-1 (HIF-1) from the small abalone Haliotis diversicolor. Gene 534, 256-264 (2014).

14. Sun, Y. et al. PI3K-AKT signaling pathway is involved in hypoxia/thermal-induced immunosuppression of small abalone Haliotis diversicolor. Fish \& Shellfish Immunology 59, 492-508 (2016).

15. Hooper, C. et al. Effects of severe heat stress on immune function, biochemistry and histopathology in farmed Australian abalone (hybrid Haliotis laevigata $\times$ Haliotis rubra). Aquaculture 432, 26-37 (2014).

16. Chu, F., La Peyre, J. F. \& Burreson, C. S. Perkinsus marinus infection and potential defense-related activities in eastern oysters, Crassostrea virginica: salinity effects. Journal of Invertebrate Pathology 62, 226-232 (1993). 
17. Ford, S. E. \& Haskin, H. H. Comparison of in vitro salinity tolerance of the oyster parasite, Haplosporidium nelsoni (MSX) and hemocytes from the host, Crassostrea virginica. Comparative Biochemistry and Physiology Part A: Physiology 90, 183-187 (1988).

18. Reid, H., Soudant, P., Lambert, C., Paillard, C. \& Birkbeck, H. Salinity effects on immune parameters of Ruditapes philippinarum challenged with Vibrio tapetis. Diseases of Aquatic Organisms 56, 249-258 (2003).

19. Mangood, S. \& Kassab, F. Influence Of Ginger (Zingiber Officinale) Supplementation Against GAMMA Rays Induced Immunosuppression In Male Rats. Isotope and Radiation Research 45, 567-576 (2013)

20. Zhao, D. et al. The immunosuppressive effects of continuous CpG ODNs stimulation in chinese mitten crab, Eriocheir sinensis. Invertebrate Survival. Journal 13, 34-43 (2016).

21. Adamo, S. A. Stress responses sculpt the insect immune system, optimizing defense in an ever-changing world. Developmental \& Comparative Immunology 66, 24-32 (2016).

22. Butt, D., Shaddick, K. \& Raftos, D. The effect of low salinity on phenoloxidase activity in the Sydney rock oyster, Saccostrea glomerata. Aquaculture 251, 159-166 (2006).

23. Sun, X., Zhou, S., Meng, F. \& Liu, S. De novo assembly and characterization of the garlic (Allium sativum) bud transcriptome by Illumina sequencing. Plant Cell Reports 31, 1823-1828 (2012).

24. Zhang, J., Chiodini, R., Badr, A. \& Zhang, G. The impact of next-generation sequencing on genomics. Journal of Genetics and Genomics 38, 95-109 (2011).

25. Franchini, P., Van der Merwe, M. \& Roodt-Wilding, R. Transcriptome characterization of the South African abalone Haliotis midae using sequencing-by-synthesis. BMC Research Notes 4, 1 (2011).

26. Wit, P. \& Palumbi, S. R. Transcriptome-wide polymorphisms of red abalone (Haliotis rufescens) reveal patterns of gene flow and local adaptation. Molecular Ecology 22, 2884-2897 (2013).

27. Shiel, B. P., Hall, N. E., Cooke, I. R., Robinson, N. A. \& Strugnell, J. M. De novo characterisation of the greenlip abalone transcriptome (Haliotis laevigata) with a focus on the heat shock protein 70 (HSP70) family. Marine Biotechnology 17, 23-32 (2015).

28. Huang, Z. et al. Pyrosequencing of Haliotis diversicolor transcriptomes: insights into early developmental molluscan gene expression. PloS One 7, e51279 (2012)

29. Bester-Van Der Merwe, A., Blaauw, S., Du Plessis, J. \& Roodt-Wilding, R. Transcriptome-wide single nucleotide polymorphisms (SNPs) for abalone (Haliotis midae): validation and application using GoldenGate medium-throughput genotyping assays. International Journal of Molecular Sciences 14, 19341-19360 (2013).

30. Valenzuela-Muñoz, V., Bueno-Ibarra, M. A. \& Escárate, C. G. Characterization of the transcriptomes of Haliotis rufescens reproductive tissues. Aquaculture Research 45, 1026-1040 (2014).

31. Picone, B., Rhode, C. \& Roodt-Wilding, R. Transcriptome profiles of wild and cultured South African abalone, Haliotis midae. Marine Genomics 20, 3-6 (2015).

32. Lim, H. J. et al. Transcriptome profiling of the Pacific oyster Crassostrea gigas by Illumina RNA-seq. Genes \& Genomics 38, 359-365 (2016).

33. Li, L. et al. Evaluation of SNPs from Transcriptome dataset in zhikong scallop Chlamys farreri. Fisheries Science 12, 007 (2012).

34. Li, G. et al. Tegillarca granosa extract Haishengsu (HSS) suppresses expression of mdr1, BCR/ABL and sorcin in drug-resistant K562/ADM tumors in mice. Advances in Medical Sciences 58, 112-117 (2013).

35. Hou, R. et al. Transcriptome sequencing and de novo analysis for Yesso scallop (Patinopecten yessoensis) using 454 GS FLX. PloS One 6, e21560 (2011).

36. Clark, M. S. et al. Insights into shell deposition in the Antarctic bivalve Laternula elliptica: gene discovery in the mantle transcriptome using 454 pyrosequencing. Bmc Genomics 11, 362 (2010).

37. Craft, J. A. et al. Pyrosequencing of Mytilus galloprovincialis cDNAs: tissue-specific expression patterns. PLoS One 5, e8875 (2010).

38. Sadamoto, H. et al. De novo sequencing and transcriptome analysis of the central nervous system of mollusc Lymnaea stagnalis by deep RNA sequencing. PloS One 7, e42546 (2012).

39. Zhang, L., Li, L., Zhu, Y., Zhang, G. \& Guo, X. Transcriptome analysis reveals a rich gene set related to innate immunity in the Eastern oyster (Crassostrea virginica). Marine Biotechnology 16, 17-33 (2014).

40. Werner, G. D., Gemmell, P., Grosser, S., Hamer, R. \& Shimeld, S. M. Analysis of a deep transcriptome from the mantle tissue of Patella vulgata Linnaeus (Mollusca: Gastropoda: Patellidae) reveals candidate biomineralising genes. Marine Biotechnology 15, 230-243 (2013).

41. Song, H. et al. De novo transcriptome sequencing and analysis of Rapana venosa from six different developmental stages using Hiseq. 2500. Comparative Biochemistry and Physiology Part D: Genomics and Proteomics 17, 48-57 (2016).

42. Zhang, X. et al. Integrative transcriptome analysis and discovery of genes involving in immune response of hypoxia/thermal challenges in the small abalone Haliotis diversicolor. Fish \& Shellfish Immunology 84, 609-626 (2019).

43. Altschul, S. F. et al. Gapped BLAST and PSI-BLAST: a new generation of protein database search programs. Nucleic Acids Research 25, 3389-3402 (1997).

44. Deng, Y. et al. Integrated nr database in protein annotation system and its localization. Comput Eng 32, 71-74 (2006).

45. Apweiler, R. et al. UniProt: the universal protein knowledgebase. Nucleic Acids Research 32, D115-D119 (2004).

46. Ashburner, M. et al. Gene Ontology: tool for the unification of biology. Nature Genetics 25, 25-29 (2000).

47. Tatusov, R. L., Galperin, M. Y., Natale, D. A. \& Koonin, E. V. The COG database: a tool for genome-scale analysis of protein functions and evolution. Nucleic Acids Research 28, 33-36 (2000).

48. Kanehisa, M., Goto, S., Kawashima, S., Okuno, Y. \& Hattori, M. The KEGG resource for deciphering the genome. Nucleic Scids. Research 32, D277-D280 (2004).

49. Iwanaga, S. \& Lee, B.-L. Recent advances in the innate immunity of invertebrate animals. BMB Reports 38, 128-150 (2005).

50. Okkenhaug, K. \& Vanhaesebroeck, B. PI3K in lymphocyte development, differentiation and activation. Nature Reviews Immunology 3, 317-330 (2003).

51. Hanna, S. \& El-Sibai, M. Signaling networks of Rho GTPases in cell motility. Cellular Signalling 25, 1955-1961 (2013).

52. Liu, Y. et al. The lipid metabolism gene FTO influences breast cancer cell energy metabolism via the PI3K/AKT signaling pathway. Oncol Lett 13, 4685-4690 (2017).

53. Jiang, W. et al. Transcriptome analysis of the Yesso scallop, Patinopecten yessoensis gills in response to water temperature fluctuations. Fish \& Shellfish Immunology 80, 133-140 (2018).

54. Wang, F. et al. ChAkt1 involvement in orchestrating the immune and heat shock responses in Crassostrea hongkongensis: molecular cloning and functional characterization. Fish \& Shellfish Immunology 47, 1015-1023 (2015).

55. Shi, Y. \& He, M. X. PfIRR interacts with HrIGF-I and activates the MAP-kinase and PI3-kinase signaling pathways to regulate glycogen metabolism in Pinctada fucata. Scientific Reports 6, 22063 (2016).

56. Dong, W., Chen, Y., Lu, W., Wu, B. \& Qi, P. Transcriptome analysis of Mytilus coruscus hemocytes in response to Vibrio alginnolyficus infection. Fish \& Shellfish Immunology 70, 560-567 (2017).

57. Zhang, M. et al. Transcriptome analysis and discovery of genes involved in immune pathways from coelomocytes of Onchidium struma after bacterial challenge. Fish \& Shellfish Immunology 72, 528-543 (2018).

58. Yu, J., Wang, H., Yue, X. \& Liu, B. Dynamic immune and metabolism response of clam Meretrix petechialis to Vibrio challenge revealed by a time series of transcriptome analysis. Fish \& Shellish Immunology 94, 17-26 (2019).

59. Fukao, T. \& Koyasu, S. PI3K and negative regulation of TLR signaling. Trends Immunol 24, 358-363 (2003). 
60. Mattioli, B., Giordani, L., Quaranta, M. G. \& Viora, M. J. Fl Leptin exerts an anti-apoptotic effect on human dendritic cells via the PI3K-Akt signaling pathway. FEBS letters 583, 1102-1106 (2009).

61. Yang, S. H., Sharrocks, A. D. \& Whitmarsh, A. J. Transcriptional regulation by the MAP kinase signaling cascades. Gene 320, 3-21 (2003).

62. Canesi, L. et al. Interactions between Mytilus haemocytes and different strains of Escherichia coli and Vibrio cholerae O1 El Tor: role of kinase-mediated signalling. Cellular Microbiology 7, 667-674 (2005).

63. Zhu, B. \& Wu, X. Identification of outer membrane protein ompR from rickettsia-like organism and induction of immune response in Crassostrea ariakensis. Molecular Immunology 45, 3198-3204 (2008).

64. Travers, M. A. et al. Pathogenic Vibrio harveyi, in contrast to non-pathogenic strains, intervenes with the p38 MAPK pathway to avoid an abalone haemocyte immune response. Journal of Cellular Biochemistry 106, 152-160 (2009).

65. Wu, X., Liu, X. \& Yu, Z. Analysis of novel immune-related genes and microsatellite markers in the transcriptome of Paphia undulata. Journal of Oceanology and Limnology 37, 1301-1316 (2019).

66. Tanguy, M., Gauthier-Clerc, S., Pellerin, J., Danger, J. M. \& Siah, A. The immune response of Mytilus edulis hemocytes exposed to Vibrio splendidus LGP32 strain: A transcriptomic attempt at identifying molecular actors. Fish \& Shellfish Immunology 74, 268-280 (2018).

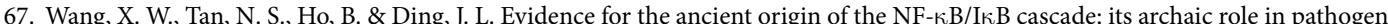
infection and immunity. Proceedings of the National Academy of Sciences of the United States of America 103, 4204-4209 (2006).

68. Baeuerle, P. A. \& Henkel, T. Function and activation of NF-kappaB in the immune system. Annual Review of Immunology 12, 141-179 (1994).

69. Sen, R. \& Baltimore, D. Multiple nuclear factors interact with the immunoglobulin enhancer sequences. Cell 46, 705-716 (1986).

70. Li, X. \& Stark, G. R. NFkB-dependent signaling pathways. Experimental Hematology 30, 285-296 (2002).

71. Detree, C., Lopez-Landavery, E., Gallardo-Escarate, C. \& Lafarga-De la Cruz, F. Transcriptome mining of immune-related genes in the muricid snail Concholepas concholepas. Fish \& Shellfish Immunology 71, 69-75 (2017).

72. Kim, B. M. et al. Transcriptome profiling suggests roles of innate immunity and digestion metabolism in purplish Washington clam. Genes. Genomics 41, 183-191 (2019).

73. Koumenis, C. et al. Regulation of p53 by hypoxia: dissociation of transcriptional repression and apoptosis from p53-dependent transactivation. Molecular and cellular biology 21, 1297-1310 (2001).

74. Jiang, Y., Loker, E. S. \& Zhang, S.-M. J. D. In vivo and in vitro knockdown of FREP2 gene expression in the snail Biomphalaria glabrata using RNA interference. Developmental \& Comparative Immunology 30, 855-866 (2006).

75. Grabherr, M. G. et al. Full-length transcriptome assembly from RNA-Seq data without a reference genome. Nature Biotechnology 29, 644-U130 (2011)

76. Mortazavi, A., Williams, B. A., McCue, K., Schaeffer, L. \& Wold, B. Mapping and quantifying mammalian transcriptomes by RNASeq. Nat Methods 5, 621-628 (2008).

77. Li, B. \& Dewey, C. N. RSEM: accurate transcript quantification from RNA-Seq data with or without a reference genome. $B M C$ Bioinformatics 12, 323 (2011).

\section{Acknowledgements}

This research was funded by National Key R\&D Program of China (Grant Number: 2018YFD0900304-5), the Natural Science Foundation of China (Grant Number: 41176152), Special Fund for Marine Economic Development of Fujian Province (Grant Number: ZHHY-2019-3), and International Science and Technology Cooperation and Communication Grant of Fujian Agriculture and Forestry University (Grant Number: KXGH17019).

\section{Author contributions}

S.Y. and X.Z. designed the study, drafted the manuscript, conducted sequence analysis and functional annotation, conducted the real-time PCR analysis, and participated in sample collection and data analysis. H.Y. and R.D. participated in data analysis and editing the manuscript. Y.W. and Z.Z. edited the manuscript and provided overall oversight of the study. All authors reviewed the manuscript.

\section{Competing interests}

The authors declare no competing interests.

\section{Additional information}

Supplementary information is available for this paper at https://doi.org/10.1038/s41598-019-56150-2.

Correspondence and requests for materials should be addressed to Y.W. or Z.Z.

Reprints and permissions information is available at www.nature.com/reprints.

Publisher's note Springer Nature remains neutral with regard to jurisdictional claims in published maps and institutional affiliations.

Open Access This article is licensed under a Creative Commons Attribution 4.0 International License, which permits use, sharing, adaptation, distribution and reproduction in any medium or format, as long as you give appropriate credit to the original author(s) and the source, provide a link to the Creative Commons license, and indicate if changes were made. The images or other third party material in this article are included in the article's Creative Commons license, unless indicated otherwise in a credit line to the material. If material is not included in the article's Creative Commons license and your intended use is not permitted by statutory regulation or exceeds the permitted use, you will need to obtain permission directly from the copyright holder. To view a copy of this license, visit http://creativecommons.org/licenses/by/4.0/.

(c) The Author(s) 2019 